\title{
Rede Neural ART Euclidiana com Treinamento Continuado
}

\author{
Angela Leite Moreno João Antonio Silva* \\ Instituto de Ciências Exatas, ICEx, UNIFAL \\ 37130-000, Alfenas, MG \\ E-mail: angela.moreno@unifal-mg.edu.br \\ E-mail: joaoa.comp@gmail.com
}

\section{Carlos Roberto Minussi}

Departamento de Engenharia Elétrica, FEIS, UNESP

15385-000, Ilha Solteira, SP

E-mail: minussi@dee.feis.unesp.br

\section{RESUMO}

Neste trabalho apresenta-se a rede neural ART Euclidiana com Treinamento Continuado, representada no fluxograma da Figura 1.

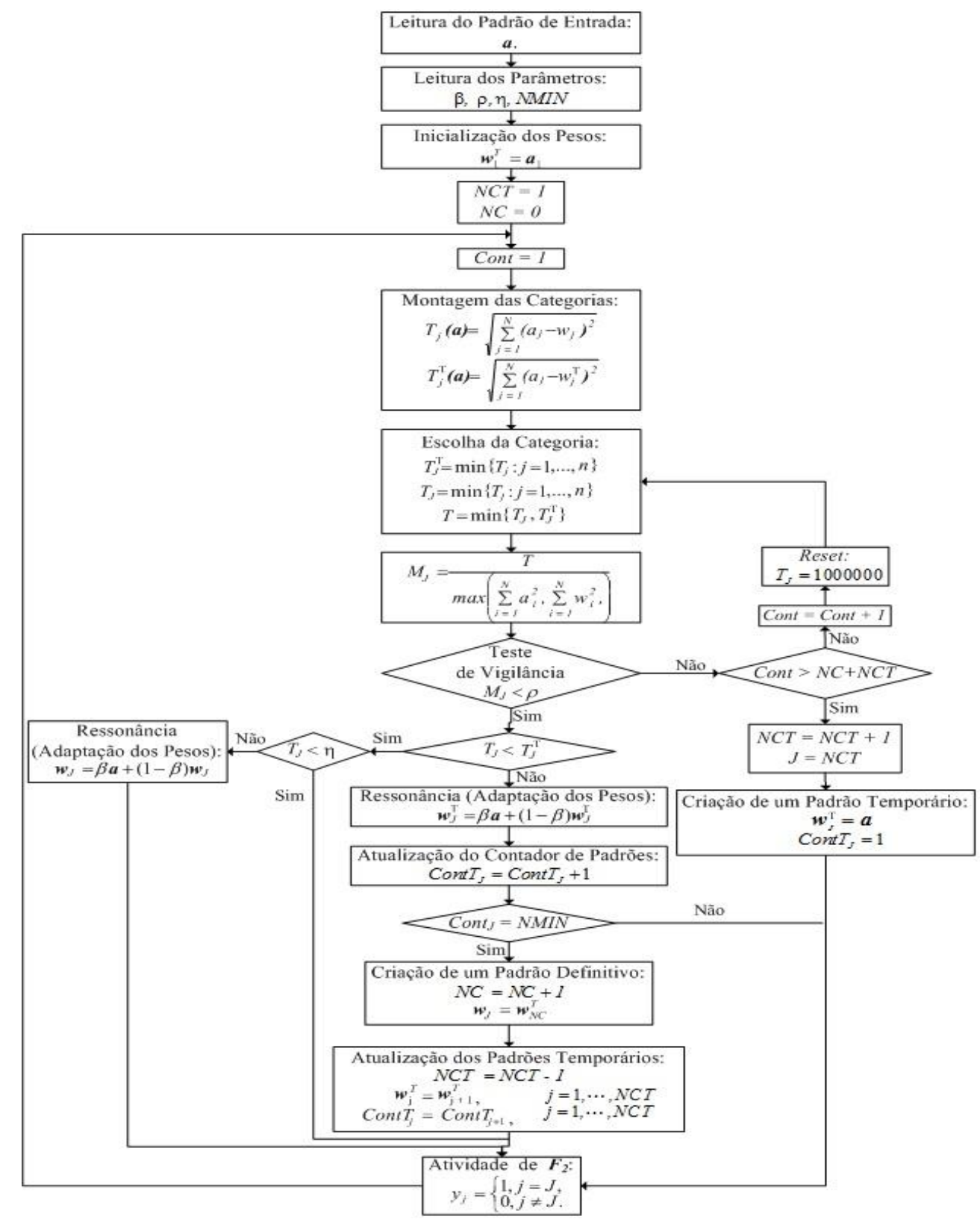

Fig. 1- Fluxograma da rede ART Euclidiana Modificada com Treinamento Continuado 
A rede ART Euclidiana com Treinamento Modificado é uma adaptação dos modelos neurais baseados na Teoria da Ressonância Adaptativa [1], mais especificamente, da Rede ART Euclidiana [2]. A Rede ART Euclidiana utiliza a distância Euclidiana entre os padrões de entrada e os centros dos clusters para classificar o padrão. E o mecanismo de treinamento continuado incorporado à rede tem o objetivo de tornar o sistema de treinamento e análise mais eficientes, de forma que a extração de conhecimento seja permanente. A primeira alteração em relação à rede neural ART Euclidiana consiste na definição de clusters temporários e clusters definitivos. Desta maneira, clusters que apresentam apenas uma anomalia não são incorporados à rede. Dois novos parâmetros também foram incorporados para se obter o mecanismo de treinamento continuado: NMIN e $\eta$. O parâmetro NMIN é responsável por dizer à rede o número de padrões necessários para que um cluster passe de temporário a definitivo. É interessante observar que a ressonância sempre ocorre em um cluster temporário. Entretanto, quando o cluster torna-se definitivo, a ressonância só ocorre se a distância entre a entrada e o centro do cluster for superior ao parâmetro $\eta$. Este segundo parâmetro, denominado índice de novidade, garante que não haja o sobre treinamento. A última adaptação é a inclusão de quatro contadores. Dois deles são responsáveis pela contagem de clusters temporários e definitivos. Um terceiro contador controla a inserção de um novo cluster temporário. E o último contador, na forma de um vetor, garante a contagem do número de padrões de entrada cuja representação é dada pelo respectivo cluster temporário.

Para validar a rede proposta foram gerados pseudo-aleatoriamente 200 pontos do plano xy com coordenadas $(\mathrm{x} ; \mathrm{y}) \epsilon[0 ; 1] \mathrm{X}[0 ; 1]$. A Figura 2 apresenta os pontos após o processo de clusterização, onde os círculos representam os pontos que pertencem a clusters definitivos enquanto que os triângulos, clusters temporários.

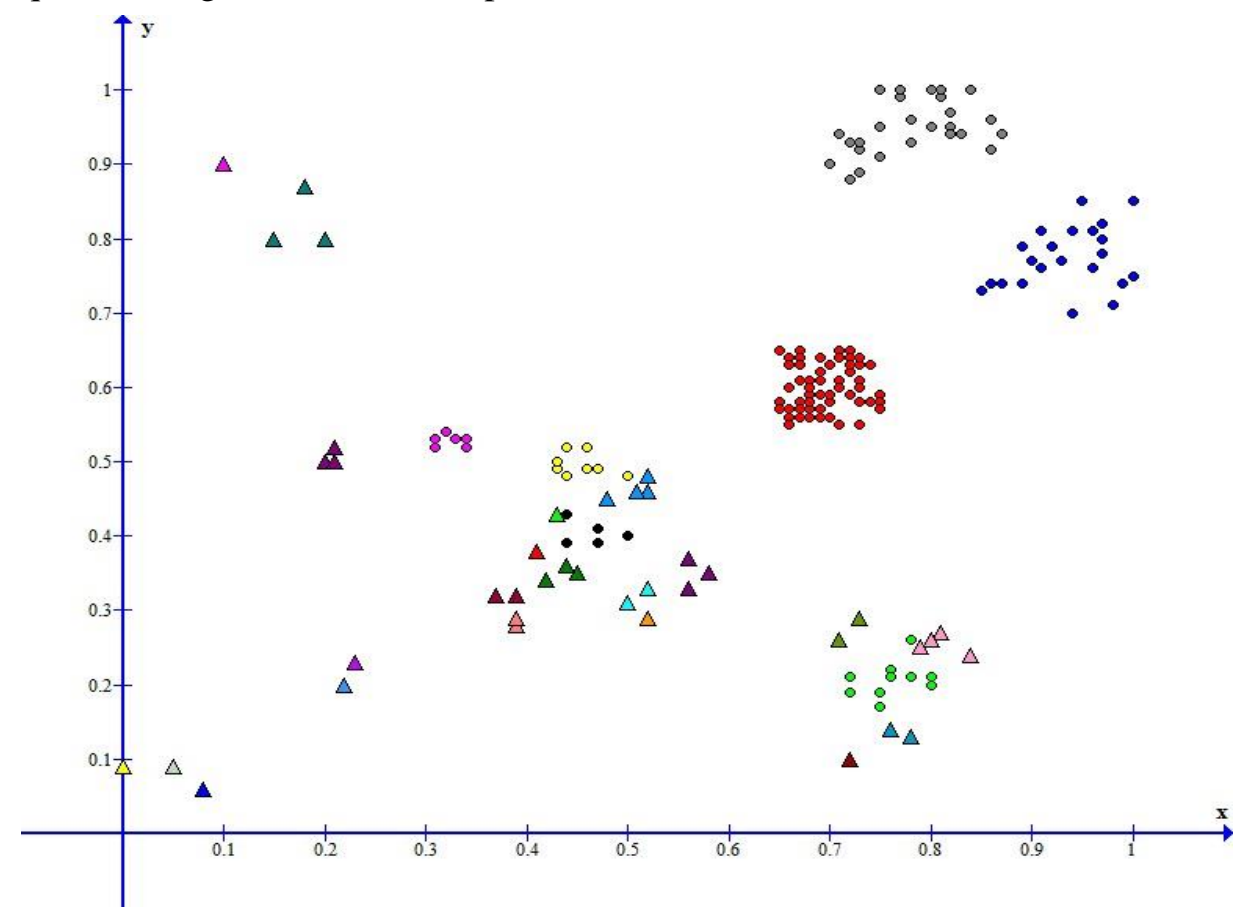

Figura 1- Pontos clusterizados pela Rede ART Euclidiana com Treinamento Continuado.

Palavras-chave: Redes Neurais Artificiais, Teoria da Ressonância Adaptativa, Treinamento Continuado.

\section{Referências}

[1] G. A. Carpenter and S. Grossberg, "A Massively Parallel Architecture for a Self-Organizing Neural Pattern Recognition Machine". Computer Vision, Graphics, and Image Processing, vol. 37, pp. 54-115, 1987.

[2] R. Kenaya, "Euclidean ART Neural Networks". In Proc 2008 World Congress on Engineering and Computer Science.

Agradecimentos à Fapemig pelo apoio financeiro. 